\title{
Renormalization-group calculation of excitation properties for impurity models
}

\author{
M. Yoshida \\ Departamento de Física, Instituto de Geociências e Ciências Exatas, Universidade Estadual Paulista, \\ 13500 Rio Claro, São Paulo, Brazil \\ M. A. Whitaker and L. N. Oliveira \\ Departamento de Física e Ciência dos Materiais, Instituto de Física e Química de São Carlos, \\ Universidade de São Paulo, 13560 São Carlos, São Paulo, Brazil
}

(Received 3 November 1989)

\begin{abstract}
The renormalization-group method developed by Wilson to calculate thermodynamical properties of dilute magnetic alloys is generalized to allow the calculation of dynamical properties of many-body impurity Hamiltonians. As a simple illustration, the impurity spectral density for the resonant-level model (i.e., the $U=0$ Anderson model) is computed. As a second illustration, for the same model, the longitudinal relaxation rate for a nuclear spin coupled to the impurity is calculated as a function of temperature.
\end{abstract}

\section{INTRODUCTION}

Strong correlations dominate the physics of dilute magnetic alloys and of fluctuating-valence and heavyfermion compounds. Analyses of such systems on the basis of single-particle concepts or straightforward perturbative expansions have long been proved unreliable, in many cases even at the qualitative level. Their theory has nevertheless advanced significantly in the past decade, driven by a combination of exact results and controlled approximations. Four techniques - the Bethe ansatz, the renormalization-group ${ }^{2}$ and Monte $\mathrm{Carlo}^{3}$ methods, and the $1 / N$ expansion ${ }^{4}$ - have played a prominent role in this development. Given that each of them has specific limitations and must be complemented by the other three, attempts to extend the scope of an individual approach are important. This paper is concerned with one such extension.

We consider the renormalization-group method, a technique originally developed to calculate thermodynamical properties of impurity models that more recently has been employed to evaluate dynamical properties of simple (essentially fixed-point) Hamiltonians. ${ }^{5}$ Nevertheless, as we shall show, to compute excitation properties away from fixed points one has to generalize the renormalization-group transformation in Ref. 2; the necessary generalization is detailed here for the first time. As an illustration of the extended method, we consider an exactly diagonalizable Hamiltonian, that of the $U=0$ spin-degenerate Anderson model ${ }^{6}$ and compute two of its dynamical properties: (1) the frequency-dependent impurity spectral density, and (2) the temperaturedependent relaxation rate for a nuclear spin coupled (by a contact interaction) to the impurity spin. Experimental motivation for the first calculation is provided by photoemission and inverse photoemission measurements, ${ }^{7}$ and for the second by NMR experiments, ${ }^{8}$ but the present work does not discuss experimental data. Our illustrative calculations are aimed at demonstrating that the novel approach solves the technical problem of calculating the excitation properties of impurity many-body Hamiltonians.

The cornerstone of the renormalization-group approach is a logarithmic discretization of the conduction band, ${ }^{2}$ which ultimately substitutes a discrete set for the continuum of eigenvalues of the model Hamiltonian. This approximation is justified $a$ posteriori: after a physical property for a given model is computed, one must show that the results converge rapidly to the continuum limit. For thermodynamical averages, this task is easily executed. ${ }^{9,10}$ For golden-rule calculations, however, a naive application of the discretization in Ref. 2 produces discrete sequences of lines bearing little resemblance to the smooth spectra expected in the continuum limit. In special cases, a rapidly convergent smoothing procedure has been shown to produce accurate results. ${ }^{5}$ That procedure nevertheless has two limitations: (1) it applies only to quadratic Hamiltonians or to Hamiltonians that, on the basis of special conservation laws, can be projected onto subspaces in which they become quadratic, and (2) it assumes that the single-particle spectrum of the model Hamiltonian is that of a conduction band with uniform phase shifts. Taken together, these two premises apply only to fixed-point Hamiltonians, in most cases limiting the computation of physical properties to restricted frequency and temperature ranges.

By contrast, the procedure in this paper applies to the entire renormalization-group flow diagram, i.e., to the fixed-point and crossover regions alike. It covers the full parametric space of many-body impurity Hamiltonians and enables us to compute physical properties for all temperatures or frequencies. Our example, the $U=0$ Anderson model, has two fixed points, both thoroughly discussed in Ref. 9: at high (low) energies, the free-orbital (frozen-impurity) fixed point dominates the physical properties of the Hamiltonian. In the renormalizationgroup approach, therefore, to calculate accurately the intermediate-energy excitation properties of even this 
quadratic Hamiltonian one must resort to the generalized procedure we describe.

Our presentation is organized as follows. Section II discusses the Hamiltonians covered by the generalized renormalization-group approach, which is detailed in Sec. III. Sections IV and V are dedicated to the two illustrations. Section VI presents our conclusions and discusses other applications of the new method. Finally, the Appendix collects an algebraic derivation too lengthy to appear in Sec. III.

\section{SCOPE OF THE METHOD}

This work concerns models comprising a single impurity orbital, denoted $c_{d}$, coupled to noninteracting $s$-wave states $c_{k}$ centered at the impurity site. The Anderson ${ }^{6}$ and the Nozières-De Dominicis ${ }^{11}$ Hamiltonians are examples. Assuming the conduction band half filled in momentum space, we measure the momenta $k$ from and in units of the Fermi momentum, so that $k$ ranges from -1 to 1 . We also assume that the interaction between conduction band and impurity is short ranged, hence momentum independent, so that the impurity couples to the conduction states through the combination $\int c_{k} d k$. It is then convenient to define the normalized Fermi operator

$$
f_{0}=\frac{1}{\sqrt{2}} \int_{-1}^{1} c_{k} d k
$$

The conduction-band-impurity interaction must be represented by a Hamiltonian involving the operators $f_{0}$ and no other combination of the $c_{k}$. Thus, in the Anderson model, the impurity and conduction states are coupled by the Hamiltonian ${ }^{9}$

$$
H_{i-c}=\sqrt{2} V\left(f_{0}^{\dagger} c_{d}+c_{d}^{\dagger} f_{0}\right),
$$

while the conduction-band and impurity Hamiltonians are

$$
H_{c}=\int_{-1}^{1} \varepsilon_{k} c_{k}^{\dagger} c_{k} d k
$$

and

$$
H_{i}=\varepsilon_{d} c_{d}^{\dagger} c_{d}+U n_{d \uparrow} n_{d \downarrow} .
$$

Following previous applications of the renormalization-group method, ${ }^{2,5,9,10}$ we consider a linear dispersion relation $\left(\varepsilon_{k}=D k\right.$, where $2 D$ is the bandwidth). The renormalization-group transformation in Sec. III nevertheless applies equally well to nonlinear relations, as a subsequent paper is planned to show. ${ }^{12}$

The analysis in Sec. III is independent of the form of the impurity Hamiltonian. $H_{i}$ involves only the impurity states $c_{d}$ and can be easily diagonalized, either numerically or analytically. The direct product of its eigenvectors with the operator $f_{0}$ [Eq. (1)] yields a basis that is complete with respect to both the impurity Hamiltonian and the impurity-conduction-band Hamiltonian $H_{i-c}$. This basis is nonetheless incomplete with respect to the conduction states and must hence be augmented. Before this can be done, however, the renormalization-group approach requires that the conduction band be discretized.
Since the discretization in this paper deviates from that in Ref. 2, its discussion deserves a separate section.

\section{FORMALISM}

\section{A. Discretization of the conduction band}

In order to make numerically tractable the model Hamiltonian in Sec. IV, we must discretize the continuum of conduction states. To this end we follow the sequence in Fig. 1, generalizing the logarithmic sequence in Ref. 2. This sequence defines intervals in momentum space. For each of them, out of the infinitely many ways in which its operators $c_{k}$ can be linearly combined, we consider the combination most localized around the impurity site. In particular, for the interval $1>k>\Lambda^{-z}$, we define the normalized Fermi operator

$$
a=\left(1-\Lambda^{-z}\right)^{-1 / 2} \int_{\Lambda^{-z}}^{1} c_{k} d k
$$

and for the interval $-1<k<-\Lambda^{-z}$ the operator

$$
b=\left(1-\Lambda^{-z}\right)^{-1 / 2} \int_{-1}^{-\Lambda^{-z}} c_{k} d k .
$$

For each of the intervals $\Lambda^{1-z-m}>k>\Lambda^{-z-m}$ $(m=1,2, \cdots)$, we likewise define the operator

$$
a_{m}=\frac{\Lambda^{(m+z-1) / 2}}{\left(1-\Lambda^{-1}\right)^{1 / 2}} \int_{\Lambda^{-z-m}}^{\Lambda^{1-z-m}} c_{k} d k
$$

and finally, for each of the intervals $-\Lambda^{1-z-m}<k$ $<-\Lambda^{-z-m}(m=1,2, \cdots)$ the operator

$$
b_{m}=\frac{\Lambda^{(m+z-1) / 2}}{\left(1-\Lambda^{-1}\right)^{1 / 2}} \int_{-\Lambda^{1-z-m}}^{-\Lambda^{-z-m}} c_{k} d k \text {. }
$$

These definitions generalize the original discretization, ${ }^{2}$ to which Fig. 1 reduces for $z=1$. We refer the reader to Refs. 2 and 9 for discussions of the approximation involved in projecting the conduction Hamiltonian $H_{c}$ on the basis $\mathcal{B}$ of the operators $a, b, a_{m}, b_{m}$, a basis clearly incomplete with respect to the operators $c_{k}$. Here, we write down the projection obtained ${ }^{13}$ by inverting Eqs. (5) $-(8)$ and substituting in Eq. (3) the resulting expressions for $c_{k}$ :

$$
\begin{aligned}
H_{c}= & \sum_{m=1}^{\infty} E_{m}(z, \Lambda)\left(a_{m}^{\dagger} a_{m}-b_{m}^{\dagger} b_{m}\right) \\
& +E_{0}(z, \Lambda)\left(a^{\dagger} a-b^{\dagger} b\right)
\end{aligned}
$$

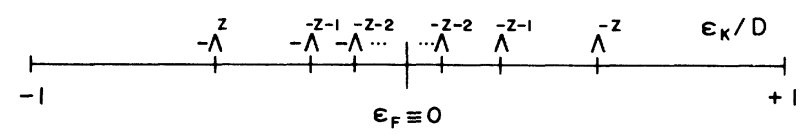

FIG. 1. Logarithmic discretization of the conduction band. The parameter $\Lambda$ must be larger than unity but is otherwise arbitrary; in numerical calculations, a typical choice is $\Lambda=3$. The parameter $z$ lies in the interval $0<z \leq 1$. For $z=1$, we recover the discretization in Refs. 2 and 9. In this case, for a linear dispersion relation, the codiagonal coefficients $\varepsilon_{n}$ on the righthand side of Eq. (13) have been determined analytically (see Ref. 2). 
where

$$
E_{m}(z, \Lambda)=\int_{\Lambda^{-z-m}}^{\Lambda^{1-z-m}} \varepsilon_{k} d k / \int_{\Lambda^{-z-m}}^{\Lambda^{1-z-m}} d k
$$

and

$$
E_{0}(z, \Lambda)=\int_{\Lambda^{-z}}^{1} \varepsilon_{k} d k / \int_{\Lambda^{-z}}^{1} d k
$$

Each energy $\pm E_{m}(z, \Lambda)$ or $\pm E_{0}(z, \Lambda)$, appearing on the right-hand side of Eq. (9), is the average kinetic energy in the corresponding intervals depicted in Fig. 1. For given $\Lambda$ and fixed $z$, these energies form a discrete set, so that, as the example in Sec. IV will show, the golden-rule calculation of photoemission rates for the discretized Hamiltonian yields discrete transition lines instead of the continuous absorption spectra one would expect. When $z$ runs from 0 to 1 , however, the energies $\pm E_{m}(z, \Lambda)$ and $\pm E_{0}(z, \Lambda)$ cover the entire conduction band, turning the golden-rule transition lines into a continuous spectrum. This smoothing procedure, detailed in Sec. IV, provides the motivation for introducing the parameter $z$ in Fig. 1.

The operator $f_{0}$, Eq. (1), can also be projected on the basis $\mathcal{B}$. By inverting Eqs. (5)-(8) we find

$$
\begin{aligned}
f_{0}= & {\left[\frac{1-\Lambda^{-1}}{2}\right]^{1 / 2} \sum_{m=1}^{\infty} \Lambda^{(1-z-m) / 2}\left(a_{m}+b_{m}\right) } \\
& +\left(\frac{1-\Lambda^{-z}}{2}\right)^{1 / 2}(a+b) .
\end{aligned}
$$

Unlike Eq. (9), this equality is exact; this can be checked by substituting the right-hand sides of Eqs. (5)-(8) for the operators $a, b, a_{m}$, and $b_{m}$, respectively. Exact, therefore, is the projection of the interaction $H_{i-c}$ [e.g., Eq. (2)] on the discrete basis $\mathcal{B}$. Only the conduction Hamiltonian is affected by the discretization in Fig. 1. This circumstance results from the definition of the operators $a, b, a_{m}$, and $b_{m}$, the first of two precautions ${ }^{2}$ taken to make the accuracy of the numerical calculations model parameter independent. The second precaution ${ }^{2}$ involves the tridiagonalization of the conduction Hamiltonian, to which we now turn.

\section{B. Tridiagonalization of the conduction Hamiltonian}

The infinite basis $\mathcal{B}$ must be made finite before the model Hamiltonian can be diagonalized numerically. At first sight, one would be tempted to truncate at finite $m$ the infinite sum on the right-hand side of Eq. (9). This must nevertheless be avoided. For then a parallel truncation on the right-hand side of Eq. (12) would be necessary, affecting the coupling between impurity and conduction electrons and making the accuracy of all subsequent computations dependent on the strength of $H_{i-c}$.

We therefore introduce a new basis. We construct an orthonormal, infinite sequence of Fermi operators $f_{n}$ ( $n=0,1,2, \cdots)$, where $f_{0}$ is the operator in Eq. (12), and the other operators are defined by the Lanczos ${ }^{14}$ construction, i.e., by requiring the conduction Hamiltonian to have the tridiagonal form

$$
H_{c}=\sum_{n=0}^{\infty} \varepsilon_{n}^{z}\left(f_{n}^{\dagger} f_{n+1}+\text { H.c. }\right) \text {. }
$$

As the Appendix shows, this condition leads to an equation determining the coefficients $\varepsilon_{n}^{z}$ :

$$
\prod_{n=0}^{N}\left(\varepsilon_{n}^{z}\right)^{2}=F_{N}(z, \Lambda)-\left[\left(\mathcal{H}_{N+1}\right)^{2 N+2}\right]_{11},
$$

where the term within the square brackets on the righthand side is the $2 N+2$ th power of the matrix

$$
\begin{aligned}
& {\left[\mathcal{H}_{N+1}\right]_{i j}=\varepsilon_{i-1}^{z} \delta_{i, j-1} }+\varepsilon_{j-1}^{z} \delta_{j, i-1}, \\
&(i, j=1,2, \cdots, N+1),
\end{aligned}
$$

and

$$
\begin{aligned}
F_{N}(z, \Lambda)= & \left(1-\Lambda^{-1}\right) \sum_{m=1}^{\infty} \Lambda^{1-z-m}\left[E_{m}(z, \Lambda)\right]^{2 N+2} \\
& +\left(1-\Lambda^{-z}\right)\left[E_{0}(z, \Lambda)\right]^{2 N+2}
\end{aligned}
$$

Equation (14) is solved iteratively. For $N=0$, it determines $\varepsilon_{0}^{z}$ :

$$
\begin{aligned}
\left(\varepsilon_{0}^{z}\right)^{2}= & \left(1-\Lambda^{-1}\right) \sum_{m=1}^{\infty} \Lambda^{1-z-m}\left[E_{m}(z, \Lambda)\right]^{2} \\
& +\left(1-\Lambda^{-z}\right)\left[E_{0}(z, \Lambda)\right]^{2} .
\end{aligned}
$$

For $N>0$, Eq. (16) determines $F_{N}(z, \Lambda)$, and provided that $\varepsilon_{0}^{z}, \varepsilon_{1}^{z}, \cdots, \varepsilon_{N-1}^{z}$ have been determined, the matrix $\left(\mathcal{H}_{N+1}\right)^{2 N+2}$ can be computed from Eq. (15). Equation (14) then determines $\varepsilon_{N}^{z}$.

For small $N(N \leq \hat{N}$, where $\hat{N}$ depends on $\Lambda$ and on the dispersion relation - a typical value is 5), this straightforward procedure can be carried out analytically or numerically. ${ }^{15}$ For larger $N$, however, a practical difficulty arises, deserving a brief digression. For increasing $n$, the coefficients $\varepsilon_{n}^{z}$ decrease rapidly; for a linear dispersion relation and $z=1$, for example, one finds ${ }^{2}$

$$
\begin{aligned}
\varepsilon_{n}^{z}=D\left(1+\Lambda^{-1}\right) \Lambda^{-n / 2} / 2 \\
\left(n \gg 1, \varepsilon_{k}=D k, \text { and } z=1\right) .
\end{aligned}
$$

As $N$ decreases, therefore, both sides of Eq. (14) become small. Since neither of the two terms on the righthand side diminishes appreciably, the small $\varepsilon_{N}^{z}$ must be computed from the difference between two relatively large numbers and for $N>\widehat{N}$ the procedure grows inaccurate.

In practice, this problem is minor. Quadrupuleprecision numerical computations make $\widehat{N} \geq 10$, and symbolic-manipulation programs make $\hat{N}$ arbitrarily large. On the other hand, as shown following, for $N \rightarrow \infty$ an asymptotic expression for the $\varepsilon_{N}^{z}$ can be easily derived. As it turns out, this expression, denoted $\hat{\varepsilon}_{N}^{z}$, describes accurately (to four significant digits, typically) the $\varepsilon_{N}^{z}$ even for $N$ as small as 10. Thus, for $N \leq \hat{N} \approx 10$ Eqs. (14) and (15) determine $\varepsilon_{N}^{z}$, while for $N>\hat{N}$ the asymptotic form $\hat{\varepsilon}_{N}^{z}$ is substituted for $\varepsilon_{N}^{z}$. The difference between $\varepsilon_{N}^{z}$ and $\hat{\varepsilon}_{N}^{z}$ (an upper bound for the error in this approximation) is insignificant. An illustration will be presented in Sec. IV $\mathrm{B}$.

In order to find the asymptotic coefficients $\hat{\varepsilon}_{N}^{z}$, we consider the conduction energies near the Fermi level. For 
these, the dispersion relation can be linearized, yielding $\varepsilon_{k} \simeq v_{F} k(k \ll 1)$, where $v_{F}$ is the Fermi velocity. Substitution in the numerator on the right-hand side of Eq. (10) then leads to

$$
E_{m}(z, \Lambda) \cong\left(\frac{v_{F} \Lambda^{1-z}}{D}\right) \frac{D\left(1+\Lambda^{-1}\right) \Lambda^{-m}}{2} \quad(m>1)
$$

The right-hand side of this equation has been factored so that the term within large parentheses on the righthand side, a constant for fixed $\Lambda$ and $z$, multiplies the discrete energies $D\left(1+\Lambda^{-1}\right) \Lambda^{-m} / 2$, precisely the energies that result ${ }^{2}$ when a linear dispersion relation is discretized for $z=1$. Since the coefficients $\varepsilon_{N}^{z}$ scale linearly with any constant factor multiplying the conduction energies $\varepsilon_{k}$, and hence with any factor multiplying the energies $E_{m}(z, \Lambda)$, the asymptotic form for $\varepsilon_{N}^{z}$ is obtained by multiplying the right-hand side of Eq. (18) by the term within large parentheses in Eq. (19):

$$
\widehat{\varepsilon}_{n}^{z}=\frac{1+\Lambda^{-1}}{2} v_{F} \Lambda^{1-z-n / 2}
$$

\section{Truncation of the conduction Hamiltonian}

With Eq. (20) and Eqs. (14) - (16), a conduction Hamiltonian with arbitrary dispersion relation can be made tridiagonal. Since $\varepsilon_{n}^{z} \rightarrow 0$ for $n \rightarrow \infty$, for given energy $\varepsilon$ one can always find an integer $N$ such that

$$
\varepsilon_{N}^{z}<\varepsilon
$$

Thus, if one is interested in calculating excitation properties at the energy $\varepsilon$ (or at the temperature $T=\varepsilon / k_{B}$, where $k_{B}$ is Boltzmann's constant), negligible error will result from truncating at $n=N$ the infinite series on the right-hand side of Eq. (13); moreover, since the conduction-band-impurity Hamiltonian $\boldsymbol{H}_{i-c}$ commutes with the operators $f_{n}(n>0)$, this error is independent of the impurity-conduction-band interaction.

With the truncated form substituted for the conduction-band Hamiltonian, the model Hamiltonian $H$ becomes

$$
H_{\text {trunc }}=H_{i}+H_{c-i}+\sum_{n=0}^{N-1} \varepsilon_{n}^{z}\left(f_{n}^{\dagger} f_{n+1}+\text { H.c. }\right) \text {. }
$$

On the right-hand side of this expression, the smallest coefficient is

$$
\varepsilon_{N-1}^{z} \approx \widehat{\varepsilon}_{N-1}^{z}=\left(1+\Lambda^{-1}\right) \Lambda^{-(N-1) / 2+1-z} v_{F} / 2
$$

[see Eq. (20)]. Following Ref. 2, to define a dimensionless Hamiltonian $H_{N}$ whose smallest coefficient is $\approx 1$, we scale $H_{\text {trunc }}$ by $1 / \widehat{\varepsilon}_{N-1}^{z}$, defining

$$
H_{N}=\frac{2}{1+\Lambda^{-1}} \Lambda^{(N-1) / 2+z-1} \frac{H_{\text {trunc }}}{v_{F}} .
$$

In the large- $N$ limit, this equality in turn defines a renormalization-group transformation $\mathcal{T}$ :

$$
\mathcal{T}\left[H_{N}\right] \equiv \sqrt{\Lambda} H_{N}+\left(f_{N}^{\dagger} f_{N+1}+\text { H.c. }\right) \approx H_{N+1} .
$$

While this transformation, essentially identical to the one discussed by Wilson, has no interesting fixed points, the conduction Hamiltonian is an important fixed point of the transformation $R \equiv \mathcal{T}^{2}$. In order to show this, that our analysis preserves the scaling ${ }^{2}$ of the conduction band, the following section discusses the specific case of a linear dispersion relation.

\section{Linear dispersion relation}

In order to apply the tridiagonalization procedure in Secs. III A and III B to the linear dispersion $\varepsilon_{k}=k D$, we must first determine the discrete energies $E_{0}(z, \Lambda)$ and $E_{m}(z, \Lambda)(m=1,2, \ldots)$. Equations (10) and (11) yield

$$
E_{m}(z, \Lambda)=D \frac{1+\Lambda^{-1}}{2} \Lambda^{1-z-m}
$$

and

$$
E_{0}(z, \Lambda)=D \frac{1+\Lambda^{-z}}{2},
$$

respectively.

TABLE I. Codiagonal coefficients for linear dispersion relation calculated for $\Lambda=3$ and $z=1$ from the numerical procedure defined by Eq. (14) $\left(\varepsilon_{n}^{z}\right)$, from the exact expression in Ref. 2 [ $\varepsilon_{n}$ (exact)], and from the asymptotic expression (20).

\begin{tabular}{rccc}
\hline \hline$n$ & $\varepsilon_{n}^{z} / D$ & $\varepsilon_{n}$ (exact) $/ D$ & $\hat{\varepsilon}_{n}^{z} / D$ \\
\hline 0 & $5.54700196 \times 10^{-1}$ & $5.54700196 \times 10^{-1}$ & $6.66666667 \times 10^{-1}$ \\
1 & $3.49370517 \times 10^{-1}$ & $3.49370517 \times 10^{-1}$ & $3.84900179 \times 10^{-1}$ \\
2 & $2.14482487 \times 10^{-1}$ & $2.14482487 \times 10^{-1}$ & $2.22222222 \times 10^{-1}$ \\
3 & $1.26748308 \times 10^{-1}$ & $1.26748308 \times 10^{-1}$ & $1.28300060 \times 10^{-1}$ \\
4 & $7.37713247 \times 10^{-2}$ & $7.37713247 \times 10^{-2}$ & $7.40740741 \times 10^{-2}$ \\
5 & $4.27081557 \times 10^{-2}$ & $4.27081557 \times 10^{-2}$ & $4.27666866 \times 10^{-2}$ \\
6 & $2.46800766 \times 10^{-2}$ & $2.46800766 \times 10^{-2}$ & $2.46913580 \times 10^{-2}$ \\
7 & $1.42533900 \times 10^{-2}$ & $1.42533900 \times 10^{-2}$ & $1.42555622 \times 10^{-2}$ \\
8 & $8.23003456 \times 10^{-3}$ & $8.23003456 \times 10^{-3}$ & $8.23045267 \times 10^{-3}$ \\
9 & $4.75177360 \times 10^{-3}$ & $4.75177360 \times 10^{-3}$ & $4.75185407 \times 10^{-3}$ \\
10 & $2.74346878 \times 10^{-3}$ & $2.74346874 \times 10^{-3}$ & $2.74348422 \times 10^{-3}$ \\
11 & $1.58794010 \times 10^{-3}$ & $1.58394837 \times 10^{-3}$ & $1.58395135 \times 10^{-3}$ \\
\hline \hline
\end{tabular}


TABLE II. Codiagonal coefficients for linear dispersion relation calculated for $\Lambda=3$ and $z=0.5$ from the numerical procedure defined by Eq. (14) $\left(\varepsilon_{n}^{z}\right)$, and from the asymptotic expression (20). The exact expression in Ref. 2 applies only to $z=1$.

\begin{tabular}{rcc}
\hline \hline$n$ & $\varepsilon_{n}^{z} / D$ & \multicolumn{1}{c}{$\hat{\varepsilon}_{n}^{z} / D$} \\
\hline 0 & $5.67544821 \times 10^{-1}$ & 1.15470054 \\
1 & $4.60312552 \times 10^{-1}$ & $6.66666667 \times 10^{-1}$ \\
2 & $4.03544580 \times 10^{-1}$ & $3.84900179 \times 10^{-1}$ \\
3 & $2.55183371 \times 10^{-1}$ & $2.22222222 \times 10^{-1}$ \\
4 & $1.35339032 \times 10^{-1}$ & $1.28300060 \times 10^{-1}$ \\
5 & $7.50818175 \times 10^{-2}$ & $7.40740741 \times 10^{-2}$ \\
6 & $4.29351236 \times 10^{-2}$ & $4.27666866 \times 10^{-2}$ \\
7 & $2.47225488 \times 10^{-2}$ & $2.46913580 \times 10^{-2}$ \\
8 & $1.42614966 \times 10^{-2}$ & $1.42555622 \times 10^{-2}$ \\
9 & $8.23159055 \times 10^{-3}$ & $8.23045267 \times 10^{-3}$ \\
10 & $4.75207278 \times 10^{-3}$ & $4.75185407 \times 10^{-3}$ \\
11 & $2.74352676 \times 10^{-3}$ & $2.74348422 \times 10^{-3}$ \\
\hline \hline
\end{tabular}

Next, we must determine the function $F_{N}(z, \Lambda)$ appearing on the right-hand side of Eq. (14). From Eq. (16) we find

$$
\begin{aligned}
F_{N}(z, \Lambda)= & \left(1-\Lambda^{-z}\right)\left[\frac{1+\Lambda^{-z}}{2}\right)^{2 N+2} \\
& +\left(1-\Lambda^{-1}\right)\left(\frac{1+\Lambda^{-1}}{2}\right)^{2 N+2} \frac{\Lambda^{-(2 N+3) z}}{1-\Lambda^{-(2 N+3)}} .
\end{aligned}
$$

Equation (14) is now solved numerically, as explained in Sec. III B. Tables I and II show codiagonal coefficients $\varepsilon_{n}^{z}$ computed in quadrupole precision for two $z$ 's (1 and 0.5 , respectively). For comparison, they also list the asymptotic values produced by Eq. (20), indicating that for $n=10$ the numerical and asymptotic $\varepsilon_{n}^{z}$ agree to four significant digits. For $n \leq 10$, on the other hand, the numerical procedure in Sec. III B yields coefficients $\varepsilon_{n}^{z}$ correct to eight significant digits; this is shown by Table I $(z=1)$, which compares them to the coefficients $\varepsilon_{n}$ given
TABLE IV. Positive eigenvalues $\hat{\eta}_{j}(j=1, \cdots, N / 2)$ of the truncated conduction Hamiltonian $H_{N}$ [Eq. (28)] for two even $N$ s (16 and 18) and two $z$ 's (1 and 0.5$)$.

\begin{tabular}{lcccc}
\hline & \multicolumn{3}{c}{$z=1$} & \multicolumn{2}{c}{$z=0.5$} \\
Eigenvalue & $N=16$ & $N=18$ & $N=16$ & $N=18$ \\
\hline$\eta_{1}$ & 1.695755 & 1.695755 & 1.695747 & 1.695752 \\
$\eta_{2}$ & 5.196104 & 5.196099 & 5.196034 & 5.196075 \\
$\eta_{3}$ & 15.58852 & 15.58848 & 15.58797 & 15.58827 \\
$\eta_{4}$ & 46.76538 & 46.76555 & 46.76527 & 46.76390 \\
$\eta_{5}$ & 140.296 & 140.2962 & 140.2961 & 140.2958 \\
$\eta_{6}$ & 420.888 & 420.8884 & 420.8883 & 420.8883 \\
$\eta_{7}$ & 1262.66 & 1262.665 & 1262.665 & 1262.665 \\
$\eta_{8}$ & 3787.99 & 3787.995 & 2587.249 & 3787.995 \\
$\eta_{9}$ & & 11363.99 & & 7761.746 \\
\hline \hline
\end{tabular}

by the exact expression in Ref. 2 . The same comparison shows that, as explained in Sec. III B, the numerical procedure determining the coefficients deteriorates rapidly for $n \geq \hat{N}=11$. Thus, by substituting the asymptotic forms $\hat{\varepsilon}_{n}^{z}$ for the $\varepsilon_{n}^{z}(n \geq 11)$, we ensure that the codiagonal coefficients of the conduction Hamiltonian deviate less than $0.1 \%$ from the exact coefficients.

Having obtained the codiagonal coefficients $\varepsilon_{n}$, we can now write and diagonalize the truncated Hamiltonian $H_{N}$. As an illustration, we consider the conduction-band Hamiltonian, i.e., we choose $H_{i}=H_{i-c}=0$. Equation (23) in this case becomes

$$
H_{N}=\Lambda^{(N-1) / 2} \sum_{n=0}^{N-1} \xi_{n}^{z}\left(f_{n}^{\dagger} f_{n+1}+\text { H.c. }\right),
$$

where

$$
\xi_{n}^{z}=\frac{2}{1+\Lambda^{-1}} \frac{\varepsilon_{n}^{z}}{D} \Lambda^{z-1}
$$

To diagonalize $H_{N}$, we write Eq. (28) in the following form:

$$
H_{N}=\sum_{m, n=0}^{N} f_{m}^{\dagger} \mathcal{H}_{m, n} f_{n},
$$

\begin{tabular}{|c|c|c|c|c|c|c|}
\hline \multirow[b]{2}{*}{ Eigenvalue } & \multicolumn{3}{|c|}{$z=1$} & \multicolumn{3}{|c|}{$z=0.5$} \\
\hline & $N=15$ & $N=17$ & $N=25$ & $N=15$ & $N=17$ & $N=25$ \\
\hline$\eta_{1}$ & 0.8000483 & 0.8000479 & 0.8000477 & 0.8000429 & 0.8000461 & 0.8000477 \\
\hline$\eta_{2}$ & 2.997492 & 2.997489 & 2.997487 & 2.997451 & 2.997475 & 2.997487 \\
\hline$\eta_{3}$ & 9.000034 & 9.000013 & 9.000000 & 8.999716 & 8.999891 & 8.999998 \\
\hline$\eta_{4}$ & 27.00001 & 27.00010 & 27.00000 & 26.99994 & 26.99915 & 26.99999 \\
\hline$\eta_{5}$ & 81.00000 & 81.00002 & 81.00001 & 81.00000 & 80.99983 & 80.99989 \\
\hline$\eta_{6}$ & 243.0000 & 243.0000 & 243.0001 & 243.0000 & 243.0000 & 242.9990 \\
\hline$\eta_{7}$ & 729.0000 & 729.0000 & 729.0011 & 729.0000 & 729.0000 & 728.9912 \\
\hline$\eta_{8}$ & 2187.000 & 2187.000 & 2187.008 & 1493.749 & 2187.000 & 2186.931 \\
\hline$\eta_{9}$ & & 6561.000 & 6561.002 & & 4481.246 & 6560.986 \\
\hline$\eta_{10}$ & & & 19683.00 & & & 19683.00 \\
\hline$\eta_{11}$ & & & 59049.00 & & & 59049.00 \\
\hline$\eta_{12}$ & & & 177147.0 & & & 177147.0 \\
\hline$\underline{\eta_{13}}$ & & & 531441.0 & & & 362981.0 \\
\hline
\end{tabular}

TABLE III. Positive eigenvalues $\eta_{1}[j=1, \cdots,(N+1) / 2]$ of the truncated conduction Hamiltonian $H_{N}$ [Eq. (28)] for three odd $N$ 's $(15,17$, and 25$)$ and two $z$ 's $(1$ and 0.5$)$. 
with

$$
\begin{array}{r}
\mathscr{H}_{m, n}=\Lambda^{(N-1) / 2}\left(\xi_{n} \delta_{m, n+1}+\xi_{m} \delta_{n, m+1}\right) \\
(m, n=0, \ldots, N) .
\end{array}
$$

The Hermitian matrix $\mathcal{H}$ can be diagonalized numerically. The results for $z=1$ and 0.5 , and for various $N$ are displayed in Tables III and IV. For large, odd $N$, the $N+1$ eigenvalues form a symmetrical set. Each positive (negative) eigenvalue is approximately given by $\eta_{j}\left(-\eta_{j}\right)$ $[j=1, \ldots,(N+1) / 2]$, where, as Table III shows,

$$
\eta_{j}=\Lambda^{j-1}[j=1, \ldots,(N-1) / 2],
$$

and

$$
\eta_{(N+1) / 2}=\frac{1+\Lambda^{-z}}{1+\Lambda^{-1}} \Lambda^{(N-1) / 2+z-1} .
$$

Likewise, for large, even $N$, the $N+1$ eigenvalues form a symmetrical set, one of them always being zero. Of the remaining ones, each positive (negative) eigenvalue is approximately given by $\hat{\eta}_{j}\left(-\hat{\eta}_{j}\right)$, where, as Table IV shows,

$$
\hat{\eta}_{j}=\Lambda^{j-1 / 2}[j=1, \cdots,(N-2) / 2],
$$

and

$$
\hat{\eta}_{N / 2}=\frac{1+\Lambda^{-z}}{1+\Lambda^{-1}} \Lambda^{(N-1) / 2+z-1} .
$$

As discussed in Refs. 2 and 9, the eigenvalue $\hat{\eta}_{0}=0$ results from the particle-hole symmetry of the conduction Hamiltonian (i.e., its invariance under the transformation $\left.c_{k} \rightarrow c_{k}^{\dagger}\right)$. Preserved by the approximations in Secs. III A-III D, this invariance makes the spectrum of $H_{c}$ symmetrical. For even $N$, the truncated Hamiltonian has an odd number $(N+1)$ of eigenvalues, so that one of them must vanish.

The results in Tables III and IV, which show that for large $N$ the scaled conduction-band Hamiltonian in Eq. (28) approaches a fixed point of the renormalizationgroup transformation $\mathcal{R}$, have a simple interpretation. ${ }^{2,9}$ In order to obtain the eigenvalues $\pm \mathscr{E}_{j}$ of the conduction Hamiltonian $H_{c}$ from those of $H_{N}$, we have to multiply the latter by $\left(1+\Lambda^{-1}\right) \Lambda^{-(N-1) / 2+1-z} v_{F} / 2$. For odd $N$, for instance, we find

$$
\begin{aligned}
\mathscr{E}_{j}=\frac{1+\Lambda^{-1}}{2} \Lambda^{-(N-1) / 2+1-z} D \eta_{j} & \\
& {[j=1, \ldots,(N+1) / 2], }
\end{aligned}
$$

or with the index $m=(N+1) / 2-j$ and Eqs. (34) and (35) substituted for $\eta_{j}$,

$$
E_{m} \equiv \mathscr{E}_{j} \approx \frac{1+\Lambda^{-1}}{2} D \Lambda^{1-z-m}[m=1, \ldots,(N-1) / 2]
$$

and

$$
E_{0} \approx \frac{1+\Lambda^{-z}}{2} D
$$

The expressions on the right-hand sides of Eqs. (37) and (38) coincide with those on the right-hand sides of Eqs. (25) and (26), respectively. For odd (even) $N$, the eigenvalues of the truncated Hamiltonian thus reproduce very well the $N+1(N)$ highest energy levels of the discretized conduction Hamiltonian. As one might expect, the central effect of the approximation in Sec. III C is to truncate the infinite series on the right-hand side of Eq. (9).

This simple illustration shows that the more general discretization in Fig. 1 preserves the essential properties of the renormalization-group approach in Ref. 2. The parameter $z$ makes that approach more flexible, as the calculation of excitation properties in Secs. IV and V will show.

\section{IMPURITY SPECTRAL DENSITY FOR THE RESONANT-LEVEL MODEL}

\section{A. Computation of the spectral density}

This section computes the impurity spectral density for the resonant-level model, a spinless Hamiltonian equivalent to the $U=0$ Anderson Hamiltonian [Eqs. $(2)-(4)]$. The resonant-level model has three characteristic energies: the band half width $D$, the impurity-orbital energy $\varepsilon_{d}$, and the half width ${ }^{6} \Gamma=\pi V^{2} / D$ of the resonance introduced in the conduction band by the coupling to the impurity states. If the latter two were zero, at low energies (i.e., for energies $\varepsilon$ much smaller than the width $D$ ) the energy-scaling invariance of the conduction band would make the Hamiltonian $H$ approach the fixed point discussed in Sec. IIID. The two characteristic energies $\varepsilon_{d}$ and $\Gamma$ break the scaling invariance and, as shown following, drive the Hamiltonian away from that fixed point.

To demonstrate this and to calculate the impurity spectral density, we first observe that for the resonant-level Hamiltonian, Eq. (23) becomes

$$
\begin{aligned}
& H_{N}=\Lambda^{(N-1) / 2}\left(\varepsilon_{d}^{z} c_{d}^{\dagger} c_{d}+V^{z}\left(f_{0}^{\dagger} c_{d}+\text { H.c. }\right)\right. \\
& \left.+\sum_{n=0}^{N-1}\left(\xi_{n}^{z} f_{n}^{\dagger} f_{n+1}+\text { H.c. }\right)\right] \text {, }
\end{aligned}
$$

where

$$
\varepsilon_{d}^{z}=\frac{\varepsilon_{d}}{D} \frac{2}{1+\Lambda^{-1}} \Lambda^{z-1},
$$

and

$$
V^{z}=\frac{V}{D} \frac{2 \sqrt{2}}{1+\Lambda^{-1}} \Lambda^{z-1}
$$

This quadratic Hamiltonian is easily diagonalized. ${ }^{9}$ For odd $N$, the following results are relevant to our analysis.

(i) Half of the $N+1$ eigenvalues of $H_{N}$ are positive, and half are negative, denoted $\eta_{j}$ and $-\eta_{j-}$, respectively, where $j=1, \ldots,(N+1) / 2$. To an excellent approximation, the following expression determines these eigenvalues: 


$$
\eta_{j \pm} \approx \Lambda^{j-1-\gamma_{J}}
$$

where

$$
\tan \left(\pi \gamma_{j \pm}\right)=\frac{\bar{\Gamma}}{\left(\varepsilon_{d}-E_{j \pm}\right)}
$$

with

$$
\begin{aligned}
& E_{j \pm}= \pm \frac{1+\Lambda^{-1}}{2} D \Lambda^{-(N-1) / 2+1-z} \eta_{j \pm} \\
& \bar{\Gamma}=\frac{\Gamma}{A_{\Lambda}},
\end{aligned}
$$

and

$$
A_{\Lambda}=\frac{1+\Lambda^{-1}}{1-\Lambda^{-1}} \frac{\ln \Lambda}{2} .
$$

(ii) The Hamiltonian is diagonalized by the Fermi operators

$$
a_{j \pm}=u_{j \pm} c_{d}+\sum_{n=0}^{N-1} u_{j n \pm} f_{n}
$$

In particular, the coefficient of the impurity operator $c_{d}$ is

$$
u_{j \pm} \approx \frac{\sin \left(\pi \gamma_{j \pm}\right)}{\left(\sin ^{2}\left(\pi \gamma_{j \pm}\right) \pm \pi \bar{\Gamma} /\left(E_{J \pm} \ln \Lambda\right)\right)^{1 / 2}}
$$

With these results, we are in a position to calculate the impurity spectral density, defined for fixed $z$ as

$$
\rho_{d}^{z}(\varepsilon)= \begin{cases}\sum_{F}\left|\left\langle F\left|c_{d}^{\dagger}\right| \Omega\right\rangle\right|^{2} \delta\left(E_{F}-E_{\Omega}-\varepsilon\right) & \text { if } \varepsilon>0 \\ \sum_{F}\left|\left\langle F\left|c_{d}\right| \Omega\right\rangle\right|^{2} \delta\left(E_{F}-E_{\Omega}-\varepsilon\right) & \text { if } \varepsilon<0,\end{cases}
$$

where $|\Omega\rangle$ and $|F\rangle$, energies $E_{\Omega}$ and $E_{F}$, are the ground state and an eigenstate of $H_{N}$, respectively. For the resonant-level Hamiltonian, the right-hand side of Eq. (49) will vanish unless, for some $j[1 \leq j \leq(N+1) / 2]$,

$$
|F\rangle=\left\{\begin{array}{lll}
a_{j+}^{\dagger}|\Omega\rangle & \text { if } & \varepsilon>0 \\
a_{j-}|\Omega\rangle & \text { if } & \varepsilon<0
\end{array}\right.
$$

so that, for $\varepsilon>0$,

$$
\left\langle F\left|c_{d}^{\dagger}\right| \Omega\right\rangle=\left\{c_{d}^{\dagger}, a_{j+}\right\}=u_{j+},
$$

while for $\varepsilon<0$,

$$
\left\langle F\left|c_{d}\right| \Omega\right\rangle=\left\{c_{d}, a_{j-}^{\dagger}\right\}=u_{j-} .
$$

In general, therefore,

$$
\rho_{d}^{z}(\varepsilon)=\sum_{j} u_{j \pm}^{2} \delta\left(\varepsilon-E_{j \pm}\right),
$$

the upper (lower) sign applying for positive (negative) $\varepsilon$.

The right-hand side of this equation, comprising a sequence of $\delta$ functions, must be smoothed to produce a continuous spectral density. To this end, we integrate $\rho_{d}^{z}$ over the discretization parameter $z$ :

$$
\rho_{d}(\varepsilon)=\int_{0}^{1} \rho_{d}^{z}(\varepsilon) d z=\int_{0}^{1} \sum_{j} u_{j \pm}^{2} \delta\left(\varepsilon-E_{j \pm}\right) d z
$$

It follows that

$$
\rho_{d}(\varepsilon)=\left.\frac{u_{j \pm}^{2}}{\left|d E_{j \pm} / d z\right|}\right|_{E_{j \pm}=\varepsilon}
$$

or substituting Eq. (48) for $u_{j \pm}$,

$$
\rho_{d}(\varepsilon)=\left.\frac{\sin ^{2}\left(\pi \gamma_{j \pm}\right)}{\left(\sin ^{2}\left(\pi \gamma_{j \pm}\right) \pm \pi \bar{\Gamma} /\left(E_{j \pm} \ln \Lambda\right)\right)\left|d E_{j \pm} / d z\right|}\right|_{E_{j \pm}=\varepsilon} .
$$

The derivative in the denominator on the right-hand side is easily computed from Eqs. (42) and (43), which yield

$$
\left|\frac{d E_{j \pm}}{d z}\right|=\frac{\pi \bar{\Gamma}}{\sin ^{2}\left(\pi \gamma_{j \pm}\right) \pm \pi \bar{\Gamma} /\left(E_{j \pm} \ln \Lambda\right)} .
$$

Equation (56) then becomes

$$
\rho_{d}(\varepsilon)=\left.\frac{\sin ^{2}\left(\pi \gamma_{j \pm}\right)}{\pi \bar{\Gamma}}\right|_{E_{J \pm}=\varepsilon} .
$$

Simple trigonometric identities applied to Eq. (43) then turn Eq. (58) into

$$
\rho_{d}(\varepsilon)=\frac{1}{\pi} \frac{\bar{\Gamma}}{\bar{\Gamma}^{2}+\left(\varepsilon_{d}-\varepsilon\right)^{2}} .
$$

For $\Lambda \rightarrow 1, A_{\Lambda}$ [Eq. (46)] converges rapidly to unity, $\bar{\Gamma}$ [Eq. (45)] converges rapidly to $\Gamma$, and Eq. (59) reduces to the exact expression ${ }^{16}$

$$
\rho_{d}(\varepsilon)=\frac{1}{\pi} \frac{\Gamma}{\Gamma^{2}+\left(\varepsilon_{d}-\varepsilon\right)^{2}} .
$$

For $\Lambda>1$, the substitution $V \rightarrow V \sqrt{A_{\Lambda}}$, a standard procedure in renormalization-group calculations, ${ }^{17}$ reduces Eq. (59) to Eq. (60).

It should be noticed that this derivation, although producing the exact expression for the spectral density, involves the errors in Eqs. (42) and (48). A numerical calculation based on the procedure in Sec. III would hence produce results in slight disagreement with Eq. (60). For $j \geq 2$, the relative errors in Eqs. (42) and (48) are nevertheless $<1 \%$; moreover, these errors diminish rapidly as $j$ increases. The renormalization-group derivation is therefore essentially exact.

\section{B. Comparison with previous renormalization-group computations}

Having derived Eq. (60), we now pause to compare the procedure in Ref. 5 with the one in Sec. III. The former approach, which amounts to convoluting the golden rule with a smoothing function, yields the following expression for the impurity spectral density:

$$
\rho_{d}(\varepsilon)=\left\{\begin{array}{l}
\sum_{F}^{\prime} \frac{\left|\left\langle F\left|c_{d}^{\dagger}\right| \Omega\right\rangle\right|^{2}}{\left(E_{F}-E_{\Omega}\right) \ln \Lambda} \text { for } \varepsilon>0 \\
\sum_{F}^{\prime} \frac{\left|\left\langle F\left|c_{d}\right| \Omega\right\rangle\right|^{2}}{\left(E_{\Omega}-E_{F}\right) \ln \Lambda} \text { for } \varepsilon<0,
\end{array}\right.
$$


where the prime restricts the sum to eigenstates $F$ of $H$ such that

$$
\frac{1}{\sqrt{\Lambda}} \leq\left|\frac{E_{F}-E_{\Omega}}{\varepsilon}\right| \leq \sqrt{\Lambda} .
$$

An analysis similar to that transforming Eq. (49) into Eq. (56) converts Eq. (61) into

$\rho_{d}(\varepsilon)=\left.\frac{\sin ^{2}\left(\pi \gamma_{j \pm}\right)}{\left(\sin ^{2}\left(\pi \gamma_{j \pm}\right) \pm \pi \bar{\Gamma} /\left(E_{j \pm} \ln \Lambda\right)\right)\left|E_{j \pm}\right| \ln \Lambda}\right|_{E_{J \pm}=\varepsilon}$

Compare now the right-hand sides of this expression and Eq. (56). Since their numerators are identical, the two fractions will be equal if and only if

$$
\left|\frac{d E_{j \pm}}{d z}\right|=\left|E_{j \pm}\right| \ln \Lambda \text {. }
$$

From Eqs. (42) and (44) it then follows that $\gamma_{j \pm}$ must be independent of $z$. As the following reasoning shows, this condition will only be satisfied if $H_{N}$ is a fixed point of the transformation $R$, defined in Sec. III D.

The eigenvalues of a fixed point $H_{N}^{*}$ of the transformation $R$ remain invariant as $N \rightarrow N+2$. For the Hamiltonian $H_{N}$ to be such a fixed point it is therefore necessary that its eigenvalues $\eta_{j \pm}-$ hence that the $\gamma_{j \pm}$ [cf. Eq. (43)] - remain invariant as $N \rightarrow N+2$, i.e., as $E_{j \pm} \rightarrow \Lambda E_{j \pm}$ [see Eq. (44)]. According to Eq. (43), this is possible under two circumstances: (i) $\Gamma=0$ and $\varepsilon_{d}=0$, or (ii) $\Gamma \rightarrow \infty$ and $\left|\varepsilon_{d}\right| \rightarrow \infty$ (or $\varepsilon_{d}=0$ ). In case (i), $\gamma_{j \pm}=0$, and we recover the (free-orbital ${ }^{9}$ ) fixed point discussed in Sec. III. In case (ii), $\pi \gamma_{j \pm}=\arctan \left(\bar{\Gamma} / \varepsilon_{d}\right)$, and we recover the (frozen-impurity ${ }^{10}$ ) fixed point.

In general, one is interested in finite parameters $\Gamma$ and $\varepsilon_{d}$, so that $H_{N}$ is no fixed point of $\mathcal{R}$. Nevertheless, for energies $\varepsilon$ such that $|\varepsilon| \gg \Gamma,\left|\varepsilon_{d}\right|$, the Hamiltonian $H_{N}$ [where Eq. (21) defines $N$ ] is close to the free-orbital fixed point. Likewise, for $|\varepsilon| \ll \Gamma,\left|\varepsilon_{d}\right|$, the Hamiltonian $H_{N}$ is close to the frozen-impurity fixed poirit. In these two ranges of energies, therefore, the properties of the model Hamiltonian are approximately described by the fixedpoint Hamiltonians, the $\gamma_{j \pm}$ are approximately independent of $z$, and Eq. (64) is a good approximation for the derivative in the denominator of $\mathrm{Eq}$. (56). In these two ranges, therefore, Eq. (63)-i.e., the procedure in Ref. 5 - can be justified.

By contrast, for $\varepsilon \approx \min \left(\Gamma,\left|\varepsilon_{d}\right|\right)$, as Eq. (43) indicates, the $\gamma_{j \pm}$ depend strongly on $E_{j \pm}$, hence on $z$, so that Eq. (64) is a poor approximation, and Eq. (56) badly underestimates the spectral density $\rho_{d}(\varepsilon)$. In this case, the generalized discretization in Fig. 1 is indispensable.

As an illustration, Fig. 2 shows the spectral densities computed from Eqs. (60) and (63) and two impurity energies, 0 and $0.05 D$. For both $\varepsilon_{d}$, for $|\varepsilon|<\Gamma=0.01 D$ the Hamiltonian $H_{N}$ [Eq. (39)] approaches the frozenimpurity fixed point, a region to which the procedure in Ref. 5 applies. Thus, in both cases, in the limit $\varepsilon \rightarrow 0$ the solid circles representing Eq. (63) agree well with the
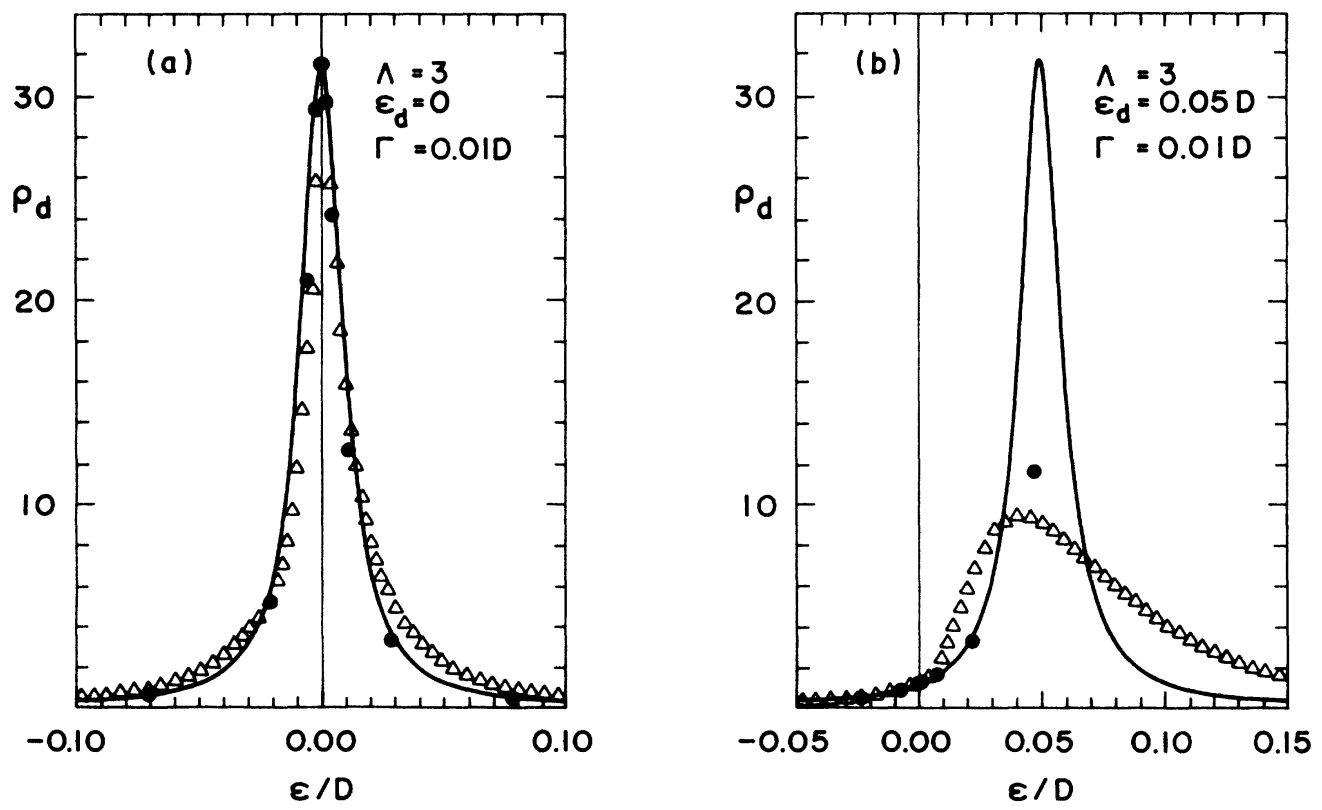

FIG. 2. $d$-level spectral densities for resonant-level width $\Gamma=0.01 D$ and two impurity energies: (a) $\varepsilon_{d}=0$ and (b) $\varepsilon_{d}=0.05 D$. The solid lines represent Eq. (60), the exact result of the procedure in Sec. III. The solid circles were obtained from Eq. (63), defined by the procedure in Ref. 5, with $\Lambda=3$; for fixed $\Lambda$, this approach yields spectral densities at discrete energies. The triangles represent Eq. (65), the Gaussian convolution in Ref. 19, for $\Lambda=3$. As explained in the text, the latter two approaches are reliable only near fixed points of the renormalization-group transformation $\mathcal{R}$, near the frozen-impurity fixed point $(|\varepsilon|\langle\Gamma)$ in particular. Thus in (a) the solid circles and triangles describe well the resonance in the spectral density at $\varepsilon \approx \varepsilon_{d}$. By contrast, in (b) Eqs. (63) and (65) give spectral densities well below the maximum of the resonance. 
solid lines representing the exact Eq. (60). In Fig. 2(a), this limit coincides with the center of the resonance, which is therefore well described by Eq. (63). By contrast, in Fig. 2(b), the maximum of the resonance lies in the crossover region so that for $\varepsilon \approx \varepsilon_{d}$ the solid circles miss the solid line by a factor of 3 - the ratio between $\left|E_{j \pm}\right| \ln \Lambda$ [which divides the right-hand side of Eq. (63)] and $\left|d E_{j \pm} d z\right|$ [which divides the right-hand side of Eq. (56)]. In this case, as pointed out previously, only the procedure in Sec. III produces accurate results.

An alternative method has been more recently proposed $^{18,19}$ to smooth the discrete spectral density resulting from Eq. (53); like the procedure in Ref. 5, this approach is accurate near the frozen-impurity fixed point. To show this, Fig. 2 displays as triangles the spectral densities obtained by convoluting Eq. (53) for $z=1$ with a Gaussian:

$$
\begin{aligned}
\rho_{d}(\varepsilon)=\int_{-\infty}^{\infty} & \frac{1}{\sqrt{\pi} \eta} \exp \left\{-\left[\frac{1}{\eta} \ln \left[\frac{\varepsilon_{j}}{\varepsilon}\right]\right]^{2}\right\} \\
& \times \sum_{j} u_{j \pm}^{2} \delta\left(\varepsilon-E_{j \pm}\right) d \ln \varepsilon .
\end{aligned}
$$

Here, as described in Ref. 19, we have carried out the integration on a logarithmic scale and taken the parameter $\eta=0.8 \ln \Lambda$. For $|\varepsilon|<\Gamma$, in both cases Eq. (65) is in good agreement with Eq. (60). In particular, in Fig. 2(a) this region contains the resonance maximum. In Fig. 2(b), however, Eq. (65) reproduces poorly the maximum in the crossover region. As one might expect, therefore, the Gaussian convolution and the convolution in Ref. 5 are comparably accurate in the fixed-point region, but describe only qualitatively the crossover region.

\section{LONGITUDINAL RELAXATION RATE}

This section considers a spin-dependent excitation property of the $U=0$ Anderson model, the longitudinal $\left(T_{1}^{-1}\right)$ relaxation rate for a spin- $\frac{1}{2}$ nucleus coupled to the impurity by the contact interaction

$$
H_{i-s}=w\left(I_{-} c_{d \uparrow}^{\dagger} c_{d \downarrow}+I_{+} c_{d \downarrow}^{\dagger} c_{d \uparrow}\right),
$$

where $w$ is a constant, and $I_{+}\left(I_{-}\right)$is the raising (lowering) nuclear-spin operator.

We wish to compute the temperature-dependent rate

$$
\frac{1}{T_{1}}=\frac{2 \pi}{Z(\beta)} \sum_{F, J} \exp \left(-\beta E_{J}\right)\left|\left\langle F\left|H_{i-s}\right| J\right\rangle\right|^{2} \delta\left(E_{F}-E_{J}\right),
$$

where $\beta=1 / k_{B} T, Z$ is the partition function, and $|J\rangle$, like $|F\rangle$, is an eigenstate of the unperturbed Hamiltonian $H_{N}$ [Eq. (39)].

To this end, we refer to the results in Sec. III but observe that the discretization in Fig. 1 is applied to the $c_{k \uparrow}$ and $c_{k \downarrow}$ separately, two independent parameters $z_{\uparrow}$ and $z_{\downarrow}$ being introduced in this spin-degenerate case. For fixed $z_{\uparrow}$ and $z_{\downarrow}$, we then compute

$$
\frac{1}{T_{i}}\left(z_{\uparrow}, z_{\downarrow}\right)=\frac{2 \pi}{Z(\beta)} \sum_{F, J} \exp \left(-\beta E_{J}\right)\left|\left\langle F\left|H_{1}\right| J\right\rangle\right|^{2} \delta\left(E_{F}-E_{J}\right)
$$

The eigenstates of $H_{N}$ split into a spin-up component and a spin-down component, e.g., $|F\rangle=|F\rangle_{\uparrow}|F\rangle_{\downarrow}$ and $E_{F}=E_{F \uparrow}+E_{F \downarrow}$. Equation (68) can therefore be written

$$
\begin{aligned}
\frac{1}{T_{1}}\left(z_{\uparrow}, z_{\downarrow}\right)=\frac{4 \pi w^{2}}{Z_{\uparrow}(\beta) Z_{\downarrow}(\beta)} \int_{-\infty}^{\infty} & \left(\sum_{F_{\uparrow}, J_{\uparrow}} \exp \left(-\beta E_{J_{\uparrow}}\right)\left|\left\langle F_{\uparrow}\left|c_{d \uparrow}^{\dagger}\right| J_{\uparrow}\right\rangle\right|^{2} \delta\left(E_{F_{\uparrow}}-E_{J_{\uparrow}}-\varepsilon\right)\right) \\
& \times\left(\sum_{F_{\downarrow}, J_{\downarrow}} \exp \left(-\beta E_{J_{\downarrow}}\right)\left|\left\langle F_{\downarrow}\left|c_{d \downarrow}\right| J_{\downarrow}\right\rangle\right|^{2} \delta\left(E_{F_{\downarrow}}-E_{J_{\downarrow}}+\varepsilon\right)\right) d \varepsilon,
\end{aligned}
$$

where a factor of 2 has appeared, accounting for the (identical) contributions from the two terms on the right-hand side of Eq. (66), and an integration over $\varepsilon$ has been introduced, to decouple the up-spin energies from the down-spin ones. Following the procedure in Sec. IV A [Eqs. (49)-(53)], we next convert the sums over the many-body states $|J\rangle$ and $|F\rangle$ into a sum over single-particle states $j$ :

$$
\frac{1}{T_{1}}\left(z_{\uparrow}, z_{\downarrow}\right)=4 \pi w^{2} \int_{-\infty}^{\infty}\left(\frac{1}{1+e^{-\beta \varepsilon}} \sum_{j} u_{j \pm}^{2} \delta\left(E_{j \pm}-\varepsilon\right) \frac{e^{-\beta \varepsilon}}{1+e^{-\beta \varepsilon}} \sum_{j} u_{j \mp}^{2} \delta\left(E_{j \mp}-\varepsilon\right)\right) d \varepsilon
$$

where the upper (lower) signs refer to $\varepsilon>0(\varepsilon<0)$.

Again following the procedure is Sec. IV A we now integrate this $z$-dependent relaxation rate, this time over $z_{\uparrow}$ and $z_{\downarrow}$, and find that

$$
\left.\frac{1}{T_{1}}=4 \pi w^{2} \int_{-\infty}^{\infty}\left|[1-f(\varepsilon)] \sum_{j} \frac{u_{j \pm}^{2}}{\left|d E_{j \pm} / d z_{\uparrow}\right|}\right| E_{J \pm}=\left.\varepsilon(\varepsilon) \sum_{j} \frac{u_{j \mp}^{2}}{\left|d E_{j \mp} / d z_{\downarrow}\right|}\right|_{E_{j \mp}=\varepsilon}\right] d \varepsilon
$$


where $f(\varepsilon)=[1+\exp (\beta \varepsilon)]^{-1}$ is the Fermi function.

Comparison with Eq. (55) now shows that each sum on the right-hand side is the impurity spectral density $\rho_{d}(\varepsilon)$, so that

$$
\frac{1}{T_{1}}=4 \pi w^{2} \int_{-\infty}^{\infty} \rho_{d}^{2}(\varepsilon)[1-f(\varepsilon)] f(\varepsilon) d \varepsilon .
$$

The renormalization-group approach based on the generalized discretization in Fig. 1 therefore reproduces the exact expression, ${ }^{20}$ applied to which standard mathematical manipulations yield the longitudinal relaxation rate. ${ }^{16}$

\section{CONCLUSIONS}

We have generalized the renormalization-group approach, ${ }^{2}$ a method developed to calculate thermodynamical properties of impurities in metallic environments; the extended method is capable of calculating excitation properties as well. More general than the convolution discussed in previous papers, ${ }^{5}$ the new procedure applies to fixed-point and crossover Hamiltonians alike. As the two illustrative applications in Secs. IV and V indicate, it calculates essentially exactly the dynamical properties as functions of energy or temperature.

To make our illustrations clear, we have calculated excitation properties for a quadratic form, the resonantlevel Hamiltonian. This has allowed us to compute the impurity spectral density and the longitudinal relaxation rate analytically. Applications to many-body Hamiltonians, such as that of the $(U \neq 0)$ Anderson model, can only be worked out numerically. The numerical diagonalization of the truncated Hamiltonian, Eq. (23), follows the iterative procedure in Refs. 2 and 9. Although not exact, this iterative diagonalization relies on a controlled approximation; ${ }^{2,9}$ the resulting deviations in the calculated physical properties can be made smaller than a few percent at any given temperature or frequency. An example based on the generalized method we have described, a numerical calculation of the impurity spectral density for the spin-degenerate Anderson model, has been reported in a preliminary publication. ${ }^{21}$

In conclusion, the discretization in Fig. 1 opens perspectives for the renormalization-group calculation of excitation properties for impurity models. The new approach may also broaden the method in a different way. In practice, the number $M$ of states that must be kept to diagonalize iteratively each model Hamiltonian limits the number of models to which the renormalization-group approach can be applied. For increasing $\Lambda, M$ decreases rapidly; the scope of the method would therefore be extended if calculations could be carried out with large $\Lambda$. Unfortunately, the computation of thermodynamical averages on the basis of the original $(z=1)$ discretization produce errors proportional to $\exp \left(-\pi^{2} / \ln \Lambda\right)$ (with large prefactors), which become serious for $\Lambda>3$.

The generalized discretization suggests an alternative approach. Our results for the thermodynamical average evaluated in Sec. V suggest that, if a given impurity $\mathrm{Ham}$ iltonian is discretized and truncated as Sec. III prescribes, then diagonalized, if thermodynamical averages (such as the impurity contribution to the magnetic susceptibility or to the specific heat) are subsequently computed and these averages integrated over $z$, then the results, such as the right-hand side of Eq. (72), should be independent of $\Lambda$. This possibility, which would pave the road for calculations with large discretization parameters, is currently being investigated, to be discussed elsewhere.

\section{ACKNOWLEDGMENTS}

This work has been partially funded by the Brazilian Financing Agency for Studies and Projects (FINEP) and the Council for Scientific and Technological Development (CNPq). Two of us (M.Y. and M.A.W.) acknowledge support from the Foundation in Support of Research in the State of São Paulo (FAPESP). M.A.W. has been additionally supported by the Coordination for the Improvement of Higher Education (CAPES).

\section{APPENDIX: CALCULATION OF CODIAGONAL COEFFICIENTS}

This appendix develops the numerical procedure converting the conduction Hamiltonian $H_{c}$ to the tridiagonal form in Eq. (13), which for convenience we write

$$
H_{c}=H_{N}+\varepsilon_{N}^{z}\left(f_{N}^{\dagger} f_{N+1}+\text { H.c. }\right)+R_{N},
$$

where

$$
H_{N}=\sum_{n=0}^{N-1} \varepsilon_{n}^{z}\left(f_{n}^{\dagger} f_{n+1}+\text { H.c. }\right),
$$

and

$$
R_{N}=\sum_{n=N+1}^{\infty} \varepsilon_{n}^{z}\left(f_{n}^{\dagger} f_{n+1}+\text { H.c. }\right),
$$

so that, for $i>N, f_{i}$ commutes with $H_{N}$, while for $i \leq N$, $f_{i}$ commutes with $R_{N}$.

We want to determine the coefficients $\varepsilon_{n}^{z}$ in Eqs. (A1) and (A2). As a preliminary step, for given integer $N$ and non-negative integer power $n<N$, we will demonstrate the following identity:

$$
f_{N}\left(H_{N}\right)^{n} f_{0}^{\dagger}|0\rangle \equiv 0 \quad(0 \leq n<N),
$$

where $|0\rangle$ denotes the state annihilated by all $c_{k}$.

The proof is recursive. For $N=1$, Eq. (A4) becomes

$$
f_{1} f_{0}^{\dagger}|0\rangle=0 \text {, }
$$

a self-evident equality, since the operators $f_{1}$ and $f_{0}$ are orthogonal.

Next, to complete the argument, we assume that

$$
f_{N-1}\left(H_{N-1}\right)^{m} f_{0}^{\dagger}|0\rangle=0 \quad(0 \leq m<N-1),
$$

and proceed to prove Eq. (A4); to this end, for $n<N$, we consider the identity

$$
\left(H_{N}\right)^{n} f_{0}^{\dagger}|0\rangle=(A+B+C)^{n} f_{0}^{\dagger}|0\rangle,
$$

where $A=H_{N-1}, B=\varepsilon_{N-1}^{z} f_{N}^{\dagger} f_{N-1}$, and $C=B^{\dagger}$.

The binomial expansion of the parentheses on the right-hand side of Eq. (A7) produces numerous terms, but 
for any integer $0 \leq m \leq n-1$ (which implies $m<N-1$ ), Eq. (A6) makes $B A^{m} f_{0}^{\dagger}|0\rangle=0$, while the equality $\left[H_{N-1}, f_{N}\right]=0$ makes $C A^{m} f_{0}^{\dagger}|0\rangle=0$. A single term $\left(A^{n}\right)$ contributes, then, to the expansion, and Eq. (A7) becomes

$$
\left(H_{N}\right)^{n} f_{0}^{\dagger}|0\rangle=\left(H_{N-1}\right)^{n} f_{0}^{\dagger}|0\rangle \quad(0 \leq n<N) .
$$

Since the operator $f_{N}$ commutes with $\left(H_{N-1}\right)^{n}$, Eq. (A4) follows.

This auxiliary relation established, we turn to providing another identity; to this end, we consider the state resulting from the repeated application of the Hamiltonian $H_{c}$ on $f_{0}^{\dagger}|0\rangle$. According to Eq. (A1), this can be written

$$
\left(H_{c}\right)^{N+1} f_{0}^{\dagger}|0\rangle=(\widetilde{A}+\widetilde{B}+\widetilde{C})^{N+1} f_{0}^{\dagger}|0\rangle \text {, }
$$

where $\tilde{A}=H_{N}, \widetilde{B}=\varepsilon_{N}^{z} f_{N+1}^{\dagger} f_{N}$, and $\widehat{C}=\widetilde{B}^{\dagger}+R_{N}$.

Again, most terms in the binomial expansion of the parentheses on the right-hand side vanish. This is because (i) for $0 \leq m<N$, according to Eq. (A4), $\widetilde{B} \widetilde{A}^{m} f_{0}^{\dagger}|0\rangle=0$, and (ii) both $f_{N+1}$ and $R_{N}$ commute with $H_{N} \equiv \widetilde{A}$, so that for any $m \geq 0, \widetilde{C} \widetilde{A}^{m} f_{0}^{\dagger}|0\rangle=0$. Thus, only two terms $\left(\widetilde{A}^{N+1}\right.$ and $\left.\widetilde{B} \widetilde{A}^{N}\right)$ contribute to the expansion, and Eq. (A9) reads

$$
\begin{aligned}
& \left(H_{c}\right)^{N+1} f_{0}^{\dagger}|0\rangle \\
& =\left(H_{N}\right)^{N+1} f_{0}^{\dagger}|0\rangle+\varepsilon_{N}^{z} f_{N+1}^{\dagger} f_{N}\left(H_{N}\right)^{N} f_{0}^{\dagger}|0\rangle .
\end{aligned}
$$

(A10)

This equality is central to the procedure determining $\varepsilon_{N}^{z}$. Before discussing that procedure, however, to refine the unwieldy last term on the right-hand side of Eq. (A10) we consider the identity (A7) for $n=N$ :

$$
\left(H_{N}\right)^{N} f_{0}^{\dagger}|0\rangle=(A+B+C)^{N} f_{0}^{\dagger}|0\rangle \text {. }
$$

Following the reasoning that converted Eq. (A9) into Eq. (A10) we convert Eq. (A11) into

$$
\begin{aligned}
\left(H_{N}\right)^{N} f_{0}^{\dagger}|0\rangle= & \left(H_{N-1}\right)^{N} f_{0}^{\dagger}|0\rangle \\
& +\varepsilon_{N-1}^{z} f_{N}^{\dagger} f_{N-1}\left(H_{N-1}\right)^{N-1} f_{0}^{\dagger}|0\rangle .
\end{aligned}
$$

Since $f_{N}$ commutes with $H_{N-1}, f_{N}$ annihilates the first term on the right-hand side. By operating with it on both sides of Eq. (A12), we then find

$f_{N}\left(H_{N}\right)^{N} f_{0}^{\dagger}|0\rangle=\varepsilon_{N-1}^{z} f_{N-1}\left(H_{N-1}\right)^{N-1} f_{0}^{\dagger}|0\rangle$.

By letting $N \rightarrow N-j(j=1, \ldots, N-1)$ on both sides of this equality, we generate $N-1$ equations which, combined with Eq. (A13), yield

$$
f_{N}\left(H_{N}\right)^{N} f_{0}^{\dagger}|0\rangle=\varepsilon_{N-1}^{z} \varepsilon_{N-2}^{z} \cdots \varepsilon_{1}^{z} f_{1} \varepsilon_{0}^{z} f_{1}^{\dagger} f_{0} f_{0}^{\dagger}|0\rangle,
$$

equivalent to

$$
f_{N}\left(H_{N}\right)^{N} f_{0}^{\dagger}|0\rangle=\prod_{n=0}^{N-1} \varepsilon_{n}^{z}|0\rangle \text {. }
$$

We now substitute this expression for $f_{N}\left(H_{N}\right)^{N} f_{0}^{\dagger}|0\rangle$ in the last term on the right-hand side of Eq. (A10) and obtain

$$
\left(H_{c}\right)^{N+1} f_{0}^{\dagger}|0\rangle=\left(H_{N}\right)^{N+1} f_{0}^{\dagger}|0\rangle+\prod_{n=0}^{N} \varepsilon_{n}^{z} f_{N+1}^{\dagger}|0\rangle
$$

Next, we calculate the norm of both sides, obtaining

$$
\begin{aligned}
\left\langle 0\left|f_{0}\left(H_{c}\right)^{2 N+2} f_{0}^{\dagger}\right| 0\right\rangle= & \left\langle 0\left|f_{0}\left(H_{N}\right)^{2 N+2} f_{0}^{\dagger}\right| 0\right\rangle \\
& +\prod_{n=0}^{N}\left(\varepsilon_{n}^{z}\right)^{2} .
\end{aligned}
$$

In order to prove this equality identical to Eq. (14), we must now find an analytical expression for the expectation value on the left-hand side of Eq. (A17), i.e., for the norm of the state $\left|\Phi_{N+1}\right\rangle \equiv\left(H_{c}\right)^{N+1} f_{0}^{\dagger}|0\rangle$. Given the identity $H_{c}|0\rangle=0$ - which follows from the definition of $|0\rangle$-by successively commuting $H_{c}$ with $f_{0}$ we find that

$$
\left|\Phi_{N+1}\right\rangle=\left[H_{c},\left[H_{c}, \cdots,\left[H_{c}, f_{0}^{\dagger}\right] \cdots\right]\right]|0\rangle .
$$

This expression, whose right-hand side nests $N+1$ commutators, effectively determines $\left|\Phi_{N+1}\right\rangle$ because an expression for the multiple commutator is easily found:

$$
\begin{aligned}
{\left[H_{c},\left[H_{c}, \cdots,\left[H_{c}, f_{0}^{\dagger}\right] \cdots\right]\right]=} & {\left[\frac{1-\Lambda^{-z}}{2}\right)^{1 / 2}\left[a^{\dagger}-(-1)^{N} b^{\dagger}\right]\left[E_{0}(z, \Lambda)\right]^{N+1} } \\
& +\left(\frac{1-\Lambda^{-1}}{2}\right)^{1 / 2} \sum_{m=1}^{\infty} \Lambda^{(1-z-m) / 2}\left[E_{m}(z, \Lambda)\right]^{N+1}\left[a_{m}^{\dagger}-(-1)^{N} b_{m}^{\dagger}\right] .
\end{aligned}
$$

The norm $\left\langle\Phi_{N+1} \mid \Phi_{N+1}\right\rangle \equiv F_{N}(z, \Lambda)$ is therefore

$$
F_{N}(z, \Lambda)=\left(1-\Lambda^{-1}\right) \sum_{m=1}^{\infty} \Lambda^{1-z-m}\left[E_{m}(z, \Lambda)\right]^{2 N+2}+\left(1-\Lambda^{-z}\right)\left[E_{0}(z, \Lambda)\right]^{2 N+2} \text {. }
$$

This expression for $F_{N}(z, \Lambda)$ is finally substituted for the left-hand side of Eq. (A17), which then yields Eq. (14).

\footnotetext{
${ }^{1}$ N. Andrei, K. Furuya, and J. H. Lowenstein, Rev. Mod. Phys. 55, 331 (1983); A. M. Tsvelick and P. B. Wiegmann, Adv. Phys. 32, 453 (1983).

${ }^{2}$ K. G. Wilson, Rev. Mod. Phys. 47, 773 (1975).
}

${ }^{3}$ J. Hirsch and R. Fye, Phys. Rev. Lett. 55, 1355 (1985).

${ }^{4}$ N. E. Bickers, Rev. Mod. Phys. 59, 845 (1987).

${ }^{5}$ L. N. Oliveira and J. W. Wilkins, Phys. Rev. B 24, 4863 (1981); D. L. Cox, H. O. Frota, L. N. Oliveira, and J. W. Wilkins, 
ibid. 32, 555 (1985); L. N. Oliveira and J. W. Wilkins, ibid. 32, 696 (1985); D. L. Cox, Ph.D. thesis, Cornell University, Ithaca, New York, 1985.

${ }^{6}$ P. W. Anderson, Phys. Rev. 124, 41 (1961).

${ }^{7}$ See, e.g., J. N. Miller, D. T. Ling, P. M. Stefan, D. L. Weissman, M. L. Shek, I. Lindau, and W. E. Spicer, Phys. Rev. B 24, 1917 (1981).

${ }^{8}$ S. L. Rudaz, J. P. Ansermet, P. K. Wang, C. P. Slichter, and J. Sinfelt, Phys. Rev. Lett. 54, 71 (1985); J. P. Ansermet, P. K. Wang, C. P. Slichter, and J. Sinfelt, Phys. Rev. B 37, 1417 (1988).

${ }^{9}$ H. R. Krishna-murthy, J. W. Wilkins, and K. G. Wilson, Phys. Rev. B 21, 1003 (1980).

${ }^{10}$ H. R. Krishna-murthy, J. W. Wilkins, and K. G. Wilson, Phys. Rev. B 21, 1044 (1980).

${ }^{11} \mathrm{P}$. Nozières and C. T. De Dominicis, Phys. Rev. 178, 1097 (1969).

${ }^{12} \mathrm{M}$. Yoshida and L. N. Oliveira (unpublished).

${ }^{13}$ Equation (9) applies to dispersion relations that are odd in the momenta measured from the Fermi level. For other dispersion relations, the positive and the negative discrete energies would be asymmetric.
${ }^{14}$ For a review, see R. Haydock, in Solid State Physics, edited by H. Ehrenreich, F. Seitz, and D. Turnbull (Academic, New York, 1980), Vol. 35, p. 215.

${ }^{15}$ That the $\varepsilon_{N}^{z}$ can be easily obtained numerically from Eqs. (14) and (16) is an important convenience of our approach, since the algebra involved in solving those equations becomes very tedious for $N>3$.

${ }^{16}$ M. Salomaa, Z. Phys. B 25, 49 (1976).

${ }^{17}$ As indicated in Ref. 5 , the discretization for the conduction band renormalizes the model parameters. In particular, in the resonant-level model the coupling $V$ is reduced to the effective value $V / \sqrt{A_{\Lambda}}$, where $A_{\Lambda}$ is defined by Eq. (46), while $\varepsilon_{d}$ is not changed. To compensate for the renormalization, the parameter $V$ entering the actual calculations is multiplied by $\sqrt{A_{\Lambda}}$.

${ }^{18}$ D. E. Heim, Phys. Rev. B 33, 5254 (1986).

${ }^{19}$ O. Sakai, Y. Shimizu, and T. Kasuya, J. Phys. Soc. Jpn. 58, 3666 (1989).

${ }^{20}$ C. P. Slichter, Principles of Magnetic Resonance (SpringerVerlag, Berlin, 1978).

${ }^{21}$ H. O. Frota and L. N. Oliveira, Phys. Rev. B 33, 7871 (1986). 